\title{
Asymptotics for conservation laws involving Lévy diffusion generators
}

\author{
by \\ Piotr Biler (Wrocław), Grzegorz Karch (Wrocław) and \\ Wojbor A. Woyczyński (Cleveland, OH)
}

Dedicated to Andrzej Krzywicki, our teacher and friend

\begin{abstract}
Let $-\mathcal{L}$ be the generator of a Lévy semigroup on $L^{1}\left(\mathbb{R}^{n}\right)$ and $f: \mathbb{R} \rightarrow \mathbb{R}^{n}$ be a nonlinearity. We study the large time asymptotic behavior of solutions of the nonlocal and nonlinear equations $u_{t}+\mathcal{L} u+\nabla \cdot f(u)=0$, analyzing their $L^{p}$-decay and two terms of their asymptotics. These equations appear as models of physical phenomena that involve anomalous diffusions such as Lévy flights.
\end{abstract}

1. Introduction. Our aim is to study the large time behavior of solutions of the Cauchy problem for a class of equations

$$
u_{t}+\mathcal{L} u+\nabla \cdot f(u)=0,
$$

called here the Lévy conservation laws, where $x \in \mathbb{R}^{n}, t \geq 0, u=u(x, t)$, $u: \mathbb{R}^{n} \times \mathbb{R}^{+} \rightarrow \mathbb{R}, f: \mathbb{R} \rightarrow \mathbb{R}^{n}$ is a nonlinear term, and $-\mathcal{L}$ is the generator of a symmetric, positivity-preserving Lévy operator semigroup $e^{-t \mathcal{L}}, t>0$, on $L^{1}\left(\mathbb{R}^{n}\right)$. The operator $\mathcal{L}$ is a pseudodifferential operator defined by the symbol $a=a(\xi) \geq 0: \widehat{\mathcal{L} v}(\xi)=a(\xi) \widehat{v}(\xi)$. The function $e^{-t a(\xi)}$ is positivedefinite, so the symbol $a(\xi)$ has the Lévy-Khinchin representation (cf. [2, Ch. I, Th. 1], or [16, Th. B.2])

$$
a(\xi)=i b \xi+q(\xi)+\int_{\mathbb{R}^{n}}\left(1-e^{-i \eta \xi}-i \eta \xi \mathbb{1}_{\{|\eta|<1\}}(\eta)\right) \Pi(d \eta) .
$$

The fundamental nature of the operator $\mathcal{L}$ is clear from the perspective of probability theory. It represents the most general form of generator of a stochastically continuous Markov process with independent and stationary increments. This fact was our basic motivation for the development of the theory presented below and in other related papers.

2000 Mathematics Subject Classification: 35K, 35B40, 35Q, 60H.

Key words and phrases: generalized Burgers equation, Lévy diffusion, asymptotics of solutions, Lévy flights, anomalous diffusion, conservation laws. 
We assume (with no loss of generality) that $b=0$, i.e., there is no drift; indeed, a shift of the $x$ variable removes the drift term $b$. The function $q(\xi)$ in (1.2) is a positive-definite quadratic form on $\mathbb{R}^{n}$; again changing the variables linearly, we can assume that $q(\xi)=|\xi|^{2}$, which corresponds to the usual Laplacian $-\Delta$ on $\mathbb{R}^{n}$ as the Gaussian part of $\mathcal{L}$. Finally, $\Pi$ is a Borel measure such that $\Pi(\{0\})=0$ and $\int_{\mathbb{R}^{n}} \min \left(1,|\eta|^{2}\right) \Pi(d \eta)<\infty$.

Equation (1.1) is supplemented with the initial condition

$$
u_{0}(x)=u(x, 0)
$$

which is assumed to be an $L^{1}\left(\mathbb{R}^{n}\right)$ function. If $u_{0}(x) \geq 0$ is positive a.e., with $\int_{\mathbb{R}^{n}} u_{0}(x) d x<\infty$, then (1.1) can model evolution of mass conserving densities $u$, i.e., for all $t>0$, one has $u(x, t) \geq 0$ and $\int_{\mathbb{R}^{n}} u(x, t) d x=$ $\int_{\mathbb{R}^{n}} u_{0}(x) d x<\infty$.

We choose a simple functional framework of the Lebesgue $L^{p}\left(\mathbb{R}^{n}\right)$ spaces. However, more general function spaces of Besov and Morrey type are also suitable for a study of the solvability and asymptotics of solutions of (1.1) (cf. [3], [8]).

The present work is motivated by various physical applications of nonlinear equations with nonlocal integro-differential or pseudodifferential diffusive terms which include, e.g., anomalous growth models of molecular interfaces involving hopping and trapping phenomena [21] and hydrodynamic models with modified diffusivity [1]. Various linear differential equations involving fractional derivatives, and their applications to statistical physics, hydrodynamics, molecular biology etc., have been discussed in, e.g., [23].

Equations (1.1) generalize and extend model equations

$$
u_{t}-u_{x x}+\sum_{j=1}^{k} a_{j}\left(-\partial^{2} / \partial x^{2}\right)^{\alpha_{j} / 2} u+f(u)_{x}=0,
$$

with $0<\alpha_{j}<2, a_{j}>0$, called one-dimensional multifractal conservation laws in [5]. The fractional power of the second derivative $\left(-\partial^{2} / \partial x^{2}\right)^{\alpha / 2}$ in (1.4) is defined via the Fourier transform by $\left(\left(-\partial^{2} / \partial x^{2}\right)^{\alpha / 2} v\right)^{\uparrow}(\xi)=|\xi|^{\alpha} \widehat{v}(\xi)$.

The generalizations of multifractal conservation laws presented in this paper go in three different directions, each extension encountering its own difficulties. First, we consider the case of arbitrary space dimension $n$. Second, a simple operator $-\partial^{2} / \partial x^{2}+\left(-\partial^{2} / \partial x^{2}\right)^{\alpha / 2}$ studied in [5] is replaced by a quite general Lévy operator $\mathcal{L}$ generating a positivity-preserving linear semigroup $e^{-t \mathcal{L}}$. Third, instead of the polynomial nonlinear term, we consider an arbitrary (sufficiently smooth) nonlinearity $f$.

The classical one-dimensional Burgers equation

$$
u_{t}-u_{x x}+\left(u^{2}\right)_{x}=0,
$$

a prototype of the whole class (1.1), is a versatile model for physical phe- 
nomena where shock formation plays an important role (cf. e.g. [27] and references therein). Importance and popularity of (1.5) lie in its simplicity, but also in the fact that the well known Hopf-Cole substitution $u=-v_{x} / v$ reduces it to the linear heat equation. This nonlinear change of variables permits an explicit description of solutions of (1.5) and explains their essentially nonlinear first order asymptotics as time goes to infinity (cf. e.g. [27] and Remark 1.3 below).

Needless to say, no analog of the Hopf-Cole formula is available for our generalized conservation laws (1.1), or (1.4), which makes their study much more difficult. Thus it was somewhat surprising that having added a new (and weaker than the second derivative) diffusive term $\left(-\partial^{2} / \partial x^{2}\right)^{\alpha / 2}$, $0<\alpha<2$, one obtained an essentially different asymptotic regime for solutions of (1.4) as compared to that for (1.5). For (1.4), the first term of the asymptotics reflects the linear behavior of $e^{-t \mathcal{L}} u_{0}$, the solution of the linearized problem $u_{t}+\mathcal{L} u=0$, and only the next term takes into account the nonlinear effects (cf. Th. 1.2 in [5]).

The Burgers equation with pure fractal diffusion in several dimensions, i.e., with $\mathcal{L}=(-\Delta)^{\alpha / 2}$ in $\mathbb{R}^{n}$, has been studied in [3]. It turned out (cf. [3, Sec. 3 and 6]) that the low order diffusion generator $(-\Delta)^{\alpha / 2}, 0<\alpha<2$, is sometimes too weak for the fractal Burgers equation to have sufficiently regular solutions. In [5] we proposed a class of one-dimensional models with mixed (Brownian and Lévy $\alpha$-stable) diffusions that retained essential features of fractal diffusion, had smooth solutions, and was found acceptable by physicists.

Another class of physically motivated equations with fractal diffusions and nonlocal nonlinearities, stemming from statistical mechanics models of interaction of diffusing particles, has been considered in [8]. Connections with probability theory, stochastic differential equations and Monte Carlotype approximations of their solutions via finite systems of interacting particles ("propagation of chaos") have been studied in the papers [15] and [4].

Also, observe that equation (1.1) can be formally interpreted as a "Fokker-Planck-Kolmogorov equation" for a "nonlinear" diffusion process in McKean's sense (see [15] and [4] for more details on the subject). Indeed, consider a Markov process $X(t), t \geq 0$, which is a solution of the stochastic differential equation

$$
\begin{aligned}
d X(t) & =d S(t)-u^{-1} f(u(X(t), t)) d t \\
X(0) & \sim u_{0}(x) d x \quad \text { in law, }
\end{aligned}
$$

where $S(t)$ is the Lévy process with generator $-\mathcal{L}$. Assuming that $X(t)$ is a unique solution of (1.6), we see that the measure-valued function $v(d x, t)=$ 
$P(X(t) \in d x)$ satisfies the weak forward equation

$$
\begin{aligned}
\frac{d}{d t}\langle v(t), \eta\rangle & =\left\langle v(t), \widetilde{\mathcal{L}}_{u(t)} \eta\right\rangle, \quad \eta \in \mathcal{S}\left(\mathbb{R}^{n}\right), \\
v(0) & =u(x, 0) d x
\end{aligned}
$$

with $\widetilde{\mathcal{L}}_{u}=-\mathcal{L}+u^{-1} f(u) \cdot \nabla$. On the other hand, $u(d x, t)=u(x, t) d x$ also solves (1.7) since

$$
\frac{d}{d t}\langle u(t), \eta\rangle=\langle-\mathcal{L} u-\nabla \cdot f(u), \eta\rangle=\left\langle u,\left(-\mathcal{L}+u^{-1} f(u) \cdot \nabla\right) \eta\right\rangle
$$

so that $v(d x, t)=u(d x, t)$ and, by uniqueness, $u$ is the density of the solution of (1.6).

The paper is organized as follows: Section 2 recalls needed facts from the theory of linear Lévy semigroups; solutions of (1.1)-(1.3) are constructed in Section 3, where their time decay is also established; Sections 4 and 5 deal with two consecutive terms of the asymptotics of solutions of (1.1).

The gist of our principal results can be summarized as follows. Suppose that the operator $\mathcal{L}$ defined in (1.2) by its symbol $a=a(\xi), \xi \in \mathbb{R}^{n}$, generates a positivity-preserving, symmetric, strongly continuous semigroup $e^{-t \mathcal{L}}$ satisfying the bound $\left\|e^{-t \mathcal{L}}\right\|_{2, \infty} \leq \min \left(c_{1} t^{-N_{1} / 4}, c_{2} t^{-N_{2} / 4}\right.$ ) for all $t>0$ (as in, e.g., the monograph [12]). Any operator $\mathcal{L}$ with $a(\xi) \sim|\xi|^{\alpha}$ for small $|\xi|$, $0<\alpha<2$, and $a(\xi) \sim|\xi|^{2}$ for large $|\xi|$, is a good example here; then $N_{1}=n<N_{2}=2 n / \alpha$. In particular, the above condition is satisfied for multifractal diffusion operators $\mathcal{L}=-\Delta+\sum_{j=1}^{k} a_{j}(-\Delta)^{\alpha_{j} / 2}, 0<\alpha_{j}<2$, $\alpha=\min _{1 \leq j \leq k} \alpha_{j}, a_{j}>0$, considered in the one-dimensional case in [5]. Now, suppose that $f: \mathbb{R} \rightarrow \mathbb{R}^{n}$ is a $C^{2}$ function with $f^{\prime}(0)=0$. Then it turns out that the solutions of the initial value problem (1.1)-(1.3) with $u_{0} \in L^{1}\left(\mathbb{R}^{n}\right) \cap L^{\infty}\left(\mathbb{R}^{n}\right)$ satisfy

(i) $\|u(t)\|_{p} \leq C t^{-n(1-1 / p) / \alpha}\left\|u_{0}\right\|_{1}$,

(ii) $\left\|u(t)-e^{-t \mathcal{L}} u_{0}\right\|_{p}=o\left(t^{-n(1-1 / p) / \alpha}\right)$,

(iii) $\left\|u(t)-e^{-t \mathcal{L}} u_{0}+F \cdot\left(\nabla e^{-t \mathcal{L}} \delta_{0}\right)\right\|_{p}=o\left(t^{-n(1-1 / p) / \alpha-1 / \alpha}\right)$, where $F=\int_{0}^{\infty} \int_{\mathbb{R}^{n}} f(u(y, \tau)) d y d \tau$, as $t \rightarrow \infty$.

Thus, roughly speaking, $u(t)$ behaves like a linear combination $u_{1}\left(e^{-t \mathcal{L}}\right)+$ $u_{2} \cdot \nabla\left(e^{-t \mathcal{L}}\right)$ of the kernels $\left(e^{-t \mathcal{L}}\right) \equiv e^{-t \mathcal{L}} \delta_{0}, \nabla\left(e^{-t \mathcal{L}}\right) \equiv \nabla\left(e^{-t \mathcal{L}} \delta_{0}\right)$ of $e^{-t \mathcal{L}}$ and $\nabla e^{-t \mathcal{L}}$, respectively, for some constants $u_{1} \in \mathbb{R}$ and $u_{2} \in \mathbb{R}^{n}$. The remainder in (ii) (resp. (iii)) is of lower order than the first (resp. the first and the second) term of the asymptotics of the solution $u(t)$.

The paper makes use of techniques more sophisticated than those utilized in [5]. As a result, the assumptions on the operators $\mathcal{L}$ could be relaxed. The tools permitting us to deal with nonlinear terms are reminiscent of those employed in papers [14], [28], [17], [5]. However, the use of a mixture of 
tools related to semigroup theory and the logarithmic Sobolev inequalities seems to be novel in the context of such nonlinear equations.

Precise formulations of the main results (i), (ii), (iii) mentioned above, with extensions and generalizations, can be found in Sections 3, 4, 5 (Theorems $3.2,3.3,4.1,5.1)$. Let us also mention that the results of this paper and $[6]$ have been announced in the note [7].

REMARK 1.1. Observe that, in general (i.e., if $\int_{\mathbb{R}^{n}} u_{0} \neq 0$ ), the estimates (i)-(iii) cannot be improved because, for the symbol $a(\xi)=|\xi|^{2}+|\xi|^{\alpha}$ and large $t$, we have $\left\|e^{-t \mathcal{L}}\right\|_{1, p} \sim\left\|e^{-t(-\Delta)^{\alpha / 2}}\right\|_{1, p}=c t^{-n(1-1 / p) / \alpha}$ (cf. [5, Cor. 2.1]). However, if $\int_{\mathbb{R}^{n}} u_{0}=0$, then $\|u(t)\|_{p}=o\left(t^{-n(1-1 / p) / \alpha}\right)$ as $t \rightarrow \infty$.

REMARK 1.2. Observe that the Brownian diffusion component of $\mathcal{L}$ does not affect the long time behavior of solutions determined by the jump component of $\mathcal{L}$. On the other hand, the Brownian diffusion contributes to the smoothness of solutions displaying an instantaneous parabolic regularization effect.

REMARK 1.3. The above asymptotic result (ii) can be reformulated as a statement about the rescaled solutions

$$
u_{\lambda}(x, t)=\lambda^{n} u\left(\lambda x, \lambda^{\alpha} t\right), \quad \lambda>0,
$$

converging to a self-similar solution $c e^{-t l(-\Delta)^{\alpha / 2}} \delta_{0}$ of the linear equation $v_{t}+l(-\Delta)^{\alpha / 2} v=0$ (with some $l>0$ ) as $\lambda$ tends to $\infty$. This holds, e.g., for multifractal diffusion operators or, more generally, for $\mathcal{L}$ satisfying $l=$ $\lim _{\xi \rightarrow 0} a(\xi) /|\xi|^{\alpha} \in(0, \infty)$, a condition stronger than the assumption (2.8) introduced below in Section 2. Note that such a result can be interpreted on the level of stochastic differential equations (1.6) as a Central Limit Theorem for rescalings of the nonlinear processes $X(t)$. Observe that assumptions on the behavior of the nonlinearity $f$ at 0 are important; see Remark 5.3.

REMARK 1.4. The asymptotics of solutions of the Cauchy problem for the Burgers equation (1.5) is described by the relation

$$
t^{(1-1 / p) / 2}\left\|u(t)-U_{M}(t)\right\|_{p} \rightarrow 0 \quad \text { as } t \rightarrow \infty,
$$

where

$$
U_{M}(x, t)=\frac{1}{\sqrt{t}} \exp \left(-x^{2} /(4 t)\right)\left(K-\int_{0}^{x / \sqrt{t}} \exp \left(-z^{2} / 4\right) d z\right)^{-1}
$$

is the "source solution" such that $\int_{\mathbb{R}} U_{M}(x, 1) d x=M=\int_{\mathbb{R}} u_{0}(x) d x$ with $K=K(M)$ (see e.g. [14]). This is what we mean when we say that the long time behavior of solutions of the classical Burgers equation is genuinely nonlinear, i.e., it is not determined by the asymptotics of the linear heat equation. 
Throughout this paper we use the notation $\|u\|_{p}$ for the Lebesgue $L^{p}\left(\mathbb{R}^{n}\right)$ norms of functions and $W^{k, p}\left(\mathbb{R}^{n}\right)$ for the Sobolev spaces. The operator norm of an operator $A$ from $L^{q}\left(\mathbb{R}^{n}\right)$ to $L^{p}\left(\mathbb{R}^{n}\right)$ is denoted by $\|A\|_{q, p}$. All the integrals are over $\mathbb{R}^{n}$ unless explicitly stated otherwise. The brackets $\langle\cdot, \cdot\rangle$ provide duality between suitable function spaces. The constants independent of solutions considered and of $t$ (but perhaps dependent on the initial values) will be denoted by the same letter $C$, even if they may vary from line to line. For a variety of facts from the standard regularity theory of parabolic type equations and interpolation inequalities we refer to [20].

2. Linear symmetric Lévy semigroups. The goal of this section is to list our assumptions and to gather needed properties of solutions of the linear Cauchy problem

$$
\begin{aligned}
& u_{t}+\mathcal{L} u=0, \\
& u(x, 0)=u_{0}(x) .
\end{aligned}
$$

Details and proofs can be found in [12], [16], while more facts and probabilistic interpretations are in [2], [9], [18], [19], and [25]. Relevant topics are also presented in [11] and [26].

Our main assumption on $\mathcal{L}$ is that $-\mathcal{L}$ generates a positivity-preserving, symmetric Lévy semigroup $e^{-t \mathcal{L}}$ of linear operators on $L^{1}\left(\mathbb{R}^{n}\right)$, and its symbol $a$ has the Lévy-Khinchin representation (1.2). The semigroup $e^{-t \mathcal{L}}$ is analytic on $L^{p}\left(\mathbb{R}^{n}\right), 1<p<\infty$ (cf. [12, Th. 1.4.2] and [26, p. 13]).

Such an $\mathcal{L}$ generates a conservative semigroup, i.e., satisfying $e^{-t \mathcal{L}} 1=1$ (note that under the assumption $(1.2), e^{-t \mathcal{L}}$ has a unique extension to constant functions; cf. also [16, Th. 2.4]). In what follows we will write

$$
\mathcal{L}=-\Delta+\mathcal{H},
$$

where $\mathcal{H}$ describes the jump component of $\mathcal{L}$, which in this paper is meant as the integral term in (1.2). The presence of a strictly positive-definite form $q$ in (1.2) implies that the semigroup $e^{-t \mathcal{L}}$ is ultracontractive, i.e., $e^{-t \mathcal{L}}$ : $L^{1}\left(\mathbb{R}^{n}\right) \rightarrow L^{\infty}\left(\mathbb{R}^{n}\right)$. Of course, the operator $\mathcal{H}$ itself may or may not generate such a semigroup; e.g., the Poisson semigroup with $a(\xi)=1-e^{-i s \xi}$ and $\Pi$ purely atomic is not ultracontractive. Note that symmetric hypercontractive $L^{2} \rightarrow L^{\infty}$ semigroups (as considered in [12]) act, by duality, from $L^{1}$ to $L^{2}$, and thus from $L^{1}$ to $L^{\infty}$ (cf. [16, Th. 6.4]). Under the above assumptions, the operators $e^{-t \mathcal{L}}, t>0$, have $L^{1}$-densities (cf. [16, Th. 6.2]), and we may write

$$
\left(e^{-t \mathcal{L}}\right)=\left(e^{t \Delta}\right) *\left(e^{-t \mathcal{H}}\right)=\left(e^{-t \mathcal{H}}\right) *\left(e^{t \Delta}\right) .
$$

In what follows we will use generalized logarithmic Sobolev inequalities (cf. [12]), Nash type inequalities (cf. [11] and [12]), and their consequences expressed in terms of the decay of semigroups. In particular, we recall the 
generalized logarithmic Sobolev inequality

$$
\int_{\mathbb{R}^{n}} v^{p} \log v \leq \varepsilon\left\langle\mathcal{L} v, v^{p-1}\right\rangle+\Gamma\|v\|_{p}^{p}+\|v\|_{p}^{p} \log \|v\|_{p}^{p},
$$

valid for each $\varepsilon>0,2 \leq p<\infty$, suitable $\Gamma=\Gamma(\varepsilon, p)$, and all $v \geq 0$, $v \in \bigcup_{t>0} e^{-t \mathcal{L}}\left(L^{1}\left(\mathbb{R}^{n}\right) \cap L^{\infty}\left(\mathbb{R}^{n}\right)\right)$ (see [12, Th. 2.2.7]).

To obtain our main results on the first and second terms of the asymptotics of solutions of the nonlinear problem (1.1) we need to assume that, for all $t>0,1 \leq p \leq \infty$, and some $0<\alpha<2$, the linear semigroup satisfies

$$
\begin{aligned}
\left\|e^{-t \mathcal{L}}\right\|_{1, p} & \leq \min \left(c_{1} t^{-n(1-1 / p) / 2}, c_{2} t^{-n(1-1 / p) / \alpha}\right), \\
\left\|\nabla e^{-t \mathcal{L}}\right\|_{1, p} & \leq \min \left(c_{1} t^{-n(1-1 / p) / 2-1 / 2}, c_{2} t^{-n(1-1 / p) / \alpha-1 / \alpha}\right) .
\end{aligned}
$$

These estimates are guaranteed, in particular, if the symbol $a$ of $\mathcal{L}$ satisfies the conditions

$$
\begin{aligned}
& 0<\liminf _{\xi \rightarrow 0} \frac{a(\xi)}{|\xi|^{\alpha}} \leq \limsup _{\xi \rightarrow 0} \frac{a(\xi)}{|\xi|^{\alpha}}<\infty, \\
& 0<\inf _{\xi} \frac{a(\xi)}{|\xi|^{2}}
\end{aligned}
$$

and if $a$ is sufficiently smooth for $\xi \neq 0$. Note that if the operator $-\mathcal{L}$ generates a symmetric Lévy semigroup, then $\lim \sup _{|\xi| \rightarrow \infty} a(\xi) /|\xi|^{2}<\infty$ by [2, I. Prop. 2(i)], and that, for the symbol $h=h(\xi) \geq 0$ of the jump part $\mathcal{H}$ of $\mathcal{L}$, the condition $\lim _{|\xi| \rightarrow \infty} h(\xi) /|\xi|^{2}=0$ holds true due to [16, (2.9)].

We also assume the condition

$$
\limsup _{|\xi| \rightarrow \infty} \frac{a(\xi)-a_{0}|\xi|^{2}}{|\xi|^{\widetilde{\alpha}}}<\infty,
$$

for some $a_{0}>0$ and $\widetilde{\alpha}<2$. Condition (2.10), where, without loss of generality, we can put $a_{0}=1$, will only be used in the proof of regularity of solutions.

REMARK 2.1. The Fourier variables representation of $\left(e^{-t \mathcal{L}}\right), \nabla\left(e^{-t \mathcal{L}}\right)$, the Hausdorff-Young inequality (cf. (5.8)), and a change of variables give the properties (2.6) and (2.7) as a consequence of (2.8)-(2.10) for $2 \leq p \leq \infty$. Similar arguments are systematically used in the proof of Theorem 5.1. The case $1 \leq p<2$ in (2.7) can be proved under some smoothness assumptions on $a$ for $\xi \neq 0$. This can be done using methods from, e.g., [24, Ch. IV, Sec. 3].

Note that if $k(\xi)=a(\xi)-l|\xi|^{\alpha}$, for some $l>0$, is a symbol of another Lévy operator $\mathcal{K}$ (as is the case of multifractal operators (2.12) below), then (2.7) for $p=1$, and then for $1<p<2$, follows in view of an analog of (2.4). 
Recall that, by [26, Prop. 2.2.2], the bound (2.6), for each $1 \leq p \leq \infty$, is equivalent to the apparently weaker condition

$$
\left\|e^{-t \mathcal{L}}\right\|_{2, \infty} \leq \min \left(c_{1} t^{-n / 4}, c_{2} t^{-n /(2 \alpha)}\right) .
$$

EXAMPLE 2.1. In applications the most frequent situation is the case when $\left\|e^{-t \mathcal{L}}\right\|_{2, \infty} \leq e^{M(t)}=c t^{-N / 4}$, where $N$ is a positive number. This corresponds, e.g., to the (Markov) semigroups generated by second order elliptic operators on $\mathbb{R}^{n}, N=n$. The decay rates (2.11) and (2.6)-(2.7) appear for the semigroups $e^{-t \mathcal{L}}$ generated by multifractal diffusion operators

$$
\mathcal{L}=-\Delta+\sum_{j=1}^{k} a_{j}(-\Delta)^{\alpha_{j} / 2}, \quad 0<\alpha_{j}<2, a_{j}>0,
$$

considered in the one-dimensional case in [5]. In this case $\alpha=\min _{1 \leq j \leq k} \alpha_{j}$, so that $-\Delta$ determines the asymptotics of the linear semigroup for small $t$, and the least $\alpha_{j}$ determines the decay for large $t$. This leads to $\beta(\varepsilon) \equiv$ $\Gamma(\varepsilon, 2) \leq c_{3}-(N / 4) \log \varepsilon$ for a constant $c_{3}$ and, by [12, Cor. 2.2.8], back to $e^{M(t)} \leq c_{4} t^{-N / 4}$. The function $\beta(\varepsilon)=\Gamma(\varepsilon, 2)$ is that from the generalized logarithmic Sobolev inequality (2.5) for $p=2$; the notation follows [12]. The latter work has other examples (Ex. 2.3.2) of semigroups satisfying (2.11).

EXAmple 2.2. For a large class of generators $\mathcal{L}$ considered in [12, Ex. 2.3.4 and 2.3.5] we have

$$
\text { either } \beta(\varepsilon) \leq c \exp \left(\varepsilon^{-\gamma}\right) \quad \text { or } \quad \beta(\varepsilon) \leq c\left(1+\varepsilon^{-\gamma}\right)
$$

for some $0<\gamma<1$. However, due to the Laplacian influence in (2.1), we always expect for our semigroup $e^{-t \mathcal{L}}$ an algebraic decay rate such as $\left\|e^{-t \mathcal{L}}\right\|_{2, \infty} \leq c t^{-n / 4}$ and $\left\|e^{-t \mathcal{L}}\right\|_{1, \infty} \leq c t^{-n / 2}$.

EXAMPLE 2.3. The semigroup corresponding to $\mathcal{H}$ with the symbol $\left(|\xi|^{2}+m^{2}\right)^{1 / 2}-m, m>0$, also satisfies $(2.11)$ with $\alpha=1$. It arises in relativistic quantum mechanics (cf. [16, Ch. 11]).

3. Existence and decay of solutions; maximum principle. By a solution of the Cauchy problem for Lévy's conservation law (1.1)-(1.3) we mean a mild solution, i.e., a function $u$ in the space $\mathcal{C}([0, T] ; \mathcal{X})$ of weakly continuous functions with values in $\mathcal{X}$, satisfying the Duhamel formula

$$
u(t)=e^{-t \mathcal{L}} u_{0}-\int_{0}^{t} \nabla \cdot e^{-(t-\tau) \mathcal{L}} f(u(\tau)) d \tau
$$

for each $t \in(0, T)$. Here $\mathcal{X}$ is a suitable Banach space selected so that $e^{-t \mathcal{L}}$ acts on it as a weakly continuous semigroup. Our preferred choice is $\mathcal{X}=$ $L^{1}\left(\mathbb{R}^{n}\right) \cap L^{\infty}\left(\mathbb{R}^{n}\right)$. The above modifies the usual definition of mild solutions which requires that $u \in C([0, T] ; \mathcal{X})$ is a strongly continuous function rather 
than just weakly continuous: this modification is needed because of rather poor properties of $e^{-t \mathcal{L}}$ on $L^{\infty}\left(\mathbb{R}^{n}\right)$ (cf. a similar situation in [3] and [5]).

TheOREM 3.1. Assume that $f \in C^{1}\left(\mathbb{R}, \mathbb{R}^{n}\right)$, and $\mathcal{L}$ is of the form (1.2) and satisfies $(2.10)$. For $u_{0} \in L^{1}\left(\mathbb{R}^{n}\right) \cap L^{\infty}\left(\mathbb{R}^{n}\right)$, there exists a unique mild solution $u \in \mathcal{C}\left([0, \infty) ; L^{1}\left(\mathbb{R}^{n}\right) \cap L^{\infty}\left(\mathbb{R}^{n}\right)\right)$ of the problem (1.1)-(1.3). This solution is regular, that is,

$$
u \in C\left((0, \infty) ; W^{2,2}\left(\mathbb{R}^{n}\right)\right) \cap C^{1}\left((0, \infty) ; L^{2}\left(\mathbb{R}^{n}\right)\right),
$$

satisfies the conservation-of-integral property

$$
\int u(x, t) d x=\int u_{0}(x) d x
$$

and the contraction property

$$
\|u(t)\|_{p} \leq\left\|u_{0}\right\|_{p}
$$

for each $p \in[1, \infty]$ and all $t>0$.

Proof. To prove the local existence result we show, as usual, that the nonlinear operator

$$
\mathcal{N}(u)(t)=e^{-t \mathcal{L}} u_{0}-\int_{0}^{t} \nabla \cdot e^{-(t-\tau) \mathcal{L}} f(u(\tau)) d \tau
$$

has a unique fixed point in the Banach space

$$
\mathcal{X}_{T}=L^{\infty}\left((0, T) ; L^{1}\left(\mathbb{R}^{n}\right) \cap L^{\infty}\left(\mathbb{R}^{n}\right)\right)
$$

equipped with the norm $\|u\|_{\mathcal{X}_{T}}=\sup _{0<t<T}\|u(t)\|_{1}+\sup _{0<t<T}\|u(t)\|_{\infty}$. Thus a local-in-time mild solution of (1.1)-(1.3) is obtained, via the Banach contraction theorem, as a fixed point of $\mathcal{N}$ in the ball $B\left(u_{0}, R\right) \equiv$ $\left\{u \in \mathcal{X}_{T}:\left\|u-u_{0}\right\|_{\mathcal{X}_{T}} \leq R\right\}$, for sufficiently large $R \geq 2\left\|u_{0}\right\|_{\mathcal{X}_{T}}$ and small $T>0$. This is an immediate consequence of the inequalities

$$
\begin{aligned}
&\|\mathcal{N}(u)\|_{\mathcal{X}_{T}} \leq\left\|u_{0}\right\|_{\mathcal{X}}+\widetilde{c}(R) T^{1 / 2}\|u\|_{\mathcal{X}_{T}}, \\
&\|\mathcal{N}(u)-\mathcal{N}(v)\|_{\mathcal{X}_{T}} \leq \widetilde{c}(R) T^{1 / 2}\|u-v\|_{\mathcal{X}_{T}},
\end{aligned}
$$

valid for any $u, v \in B\left(u_{0}, R\right)$. Here, $\widetilde{c}(R)=\sup _{|s| \leq R}\left|f^{\prime}(s)\right|$, and we used crude $L^{1}$-bounds $\left\|e^{-t \mathcal{L}}\right\|_{1,1} \leq 1,\left\|\nabla e^{-t \mathcal{L}}\right\|_{1,1} \leq\left\|\nabla e^{t \Delta}\right\|_{1,1} \leq C t^{-1 / 2}$ for the linear semigroup $e^{-t \mathcal{L}}(\mathrm{cf} .(2.4))$.

The remainder of the proof depends on the fact that the solutions $u$ are regular, and the latter follows from the classical regularity result for parabolic equations which allows us to prove that

$$
u \in C\left((0, T) ; W^{2,2}\left(\mathbb{R}^{n}\right)\right) \cap C^{1}\left((0, T) ; L^{2}\left(\mathbb{R}^{n}\right)\right) .
$$

Indeed, by $(2.10)$, we have $u_{t}-\Delta u=-\mathcal{H} u-\nabla \cdot f(u) \in W^{-\widetilde{\alpha}, 2}\left(\mathbb{R}^{n}\right) \cap$ $W^{-1,2}\left(\mathbb{R}^{n}\right)$, so by a repeated use of [20, Chapter 3$]$, the regularity of solutions follows. In particular, later on, this regularity enables us to use various a priori estimates for mild solutions (as was done in [20] for weak solutions). 
Weak continuity of $u(t)$ at $t=0$ is a standard consequence of regularity of $u$ and properties of $e^{-t \mathcal{L}}$ on $\mathcal{X}$.

Now, the local solution may be extended to a global solution in view of the a priori estimate

$$
\sup _{t \in\left[0, T_{*}\right)}\left(\|u(t)\|_{1}+\|u(t)\|_{\infty}\right)<\infty
$$

where $T_{*}$ is the maximal time of existence of $u(t)$. The relation (3.4) is a straightforward corollary to (3.3) for $p=1$ and $p=\infty$. For $p=1$, an even stronger estimate (3.6) will be proved. Finally, the case $p=\infty$ is a consequence of Proposition 3.1 proved below.

Inequality (3.3) for $1<p<\infty$ is obtained by multiplying (1.1) by $|u|^{p-1} \operatorname{sgn} u$ and integrating over $\mathbb{R}^{n}$ with respect to $x$. This leads to

$$
\frac{1}{p} \frac{d}{d t} \int|u(x, t)|^{p} d x+\left\langle\mathcal{L} u,|u|^{p-1} \operatorname{sgn} u\right\rangle=0 .
$$

The second term on the left hand side of the above formula is positive in view of the inequality

$$
\frac{4(p-1)}{p^{2}}\left\langle\mathcal{L}|u|^{p / 2},|u|^{p / 2}\right\rangle \leq\left\langle\mathcal{L} u,|u|^{p-1} \operatorname{sgn} u\right\rangle,
$$

which was obtained in [26, proof of Prop. II.5.4] (cf. also [12, (2.2.7)] for $p \geq 2$ ). Hence $(d / d t)\|u(t)\|_{p}^{p} \leq 0$ and, as a consequence, (3.3) holds true for each $p \in(1, \infty)$.

The integral $\int u(x, t) d x$ is a conserved quantity for solutions of (1.1) constructed above. Indeed, integrating (1.1) over $\mathbb{R}^{n}$ with respect to $x$ we obtain $(d / d t) \int u(x, t) d x=0$, since $\int \nabla \cdot f(u(x, t)) d x=0$ and

$$
\int \mathcal{L} u(x, t) d x=\widehat{\mathcal{L} u}(0, t)=a(0) \widehat{u}(0, t)=0 .
$$

Hence, we have $\|u(t)\|_{1}=\left\|u_{0}\right\|_{1}$ for the initial data $u_{0}$ of constant sign.

The uniqueness of solutions and the inequality (3.3) for $p=1$ follow from the estimate

$$
\|u(t)-v(t)\|_{1} \leq\left\|u_{0}-v_{0}\right\|_{1}
$$

valid for arbitrary two solutions $u_{0}, v_{0}$ of (1.1)-(1.3) with $u_{0}, v_{0} \in L^{1}\left(\mathbb{R}^{n}\right)$. Its proof is obtained by multiplying the difference of equations (1.1) written for $u$ and $v$ by $\operatorname{sgn}(u-v)$ and integrating over $\mathbb{R}^{n}$. Then we get

$$
\frac{d}{d t}\|u-v\|_{1}+\langle\mathcal{L}(u-v), \operatorname{sgn}(u-v)\rangle+\int \nabla \cdot(f(u)-f(v)) \operatorname{sgn}(u-v)=0,
$$

and $(d / d t)\|u-v\|_{1} \leq 0$ results because the second term is nonnegative (cf. e.g. $[3,(3.5)])$, and the third term vanishes. The latter fact follows by a standard approximation of the sign function by smooth functions (cf. [3, Th. 3.1], [14, (2.28)]). 
REMARK 3.1. The existence, uniqueness and regularity results obtained in Theorem 3.1 for the initial conditions in $L^{1}\left(\mathbb{R}^{n}\right) \cap L^{\infty}\left(\mathbb{R}^{n}\right)$ can be easily extended to the case of $u_{0} \in L^{1}\left(\mathbb{R}^{n}\right)$, using techniques similar to those employed in [14]. First, an arbitrary $u_{0} \in L^{1}\left(\mathbb{R}^{n}\right)$ can be approximated by bounded truncations of $u_{0}$, and then a passage to the limit, using an application of (3.6) and the estimate (3.15) (for each $t>0$ ) derived below, provides a mild solution of (1.1) with $u_{0}$ as the initial condition.

Next, we establish the maximum and minimum principles for the nonlinear problem (1.1)-(1.3).

Proposition 3.1. Solutions of (1.1)-(1.3) with $u_{0} \in L^{1}\left(\mathbb{R}^{n}\right) \cap L^{\infty}\left(\mathbb{R}^{n}\right)$ satisfy the inequalities

$$
\operatorname{ess} \inf u_{0} \leq u(x, t) \leq \operatorname{ess} \sup u_{0} \quad \text { for a.e. } x, t .
$$

Proof. We show the maximum principle only, because the proof of the minimum principle is completely analogous. The reasoning uses a crucial property of $\mathcal{L}$ which is a Dirichlet operator (cf. [16] or [9]). Let $\mu=\operatorname{ess} \sup u_{0}$ and consider the function $g=(u-\mu-\varepsilon)_{+} \equiv \max (u-\mu-\varepsilon, 0)$ for an arbitrary $\varepsilon>0$. The equation (1.1) multiplied by $g$ leads to

$$
\int u_{t} g+\int g \mathcal{L} u+\int g \nabla \cdot f(u)=0,
$$

and since $\int u_{t} g=\int g_{t} g, \int g \nabla \cdot f(u)=0$, we obtain

$$
\int g^{2}(x, t) d x+\int_{0}^{t}\langle\mathcal{L} u, g\rangle=0 .
$$

The inequality $\langle\mathcal{L} u, g\rangle \geq 0$ is satisfied for (self-adjoint) generators $-\mathcal{L}$ of symmetric (sub-)Markovian semigroups (cf. [16, (3.9)], or [9, Théorème 1.1]), so $\|g\|_{2}=0$ follows. Of course, that means $u \leq \mu$ a.e., since $\varepsilon>0$ was an arbitrary number.

Solutions of (1.1) also satisfy the following comparison principle.

Corollary 3.1. Let $u$ and $v$ be solutions of the Cauchy problem (1.1)(1.3) with initial values $u_{0}, v_{0} \in L^{1}\left(\mathbb{R}^{n}\right)$, respectively. If $u_{0}(x) \leq v_{0}(x)$ a.e. then, for each $t>0, u(x, t) \leq v(x, t)$ a.e.

Proof. The nonlinear semigroup $u_{0} \mapsto u(t)$ is an $L^{1}$-contraction (cf. (3.6)) and preserves the integrals (cf. (3.2)). Since $s_{+}=(|s|+s) / 2$, the inequality $u_{0} \leq v_{0}$ a.e. gives

$$
\begin{aligned}
2 \int(u(t)-v(t))_{+} & =\int|u(t)-v(t)|+\int(u(t)-v(t)) \\
& \leq \int\left|u_{0}-v_{0}\right|+\int\left(u_{0}-v_{0}\right)=2 \int\left(u_{0}-v_{0}\right)_{+}=0,
\end{aligned}
$$

i.e., $u(t) \leq v(t)$ a.e. Thus, the nonlinear semigroup is order-preserving. 
The results presented in the remainder of this section concern the $L^{p}\left(\mathbb{R}^{n}\right)$ decay of positive solutions of (1.1)-(1.3) constructed in the first part of Theorem 3.1. First we consider the case of $p=2$. The estimate of solutions of the nonlinear equation, which turns out to be the same as for the linear semigroup, can be proved under quite general assumptions on the decay of the semigroup.

THEOREM 3.2. If the semigroup $e^{-t \mathcal{L}}$ satisfies the estimate $\left\|e^{-t \mathcal{L}}\right\|_{1, \infty} \leq$ $m(t)$ for some decreasing $C^{1}$ function $m: \mathbb{R}^{+} \rightarrow \mathbb{R}^{+}$, then positive solutions of the Cauchy problem (1.1)-(1.3) satisfy the bound

$$
\|u(t)\|_{2} \leq m(t)^{1 / 2}\left\|u_{0}\right\|_{1} .
$$

Proof. Multiplying (1.1) by $u$ we obtain

$$
\frac{d}{d t}\|u\|_{2}^{2}+\langle\mathcal{L} u, u\rangle=0
$$

since the integral $\int u \nabla \cdot f(u)$ vanishes.

Now let us recall from [11, Prop. II.1 and Th. II.5] that the decay estimate for the semigroup

$$
\left\|e^{-t \mathcal{L}}\right\|_{1, \infty} \leq m(t)
$$

for some function $m$ such that $m^{\prime}<0$, is equivalent to the generalized Nash inequality

$$
\vartheta\left(\|v\|_{2}^{2}\right) \leq\langle\mathcal{L} v, v\rangle,
$$

valid for all $v \in \mathcal{D}(\mathcal{L}),\|v\|_{1}=1$, whenever $-m^{\prime}(s)=\vartheta(m(s))$. Setting $w(t)=\|u(t)\|_{2}^{2}$ and $K=\|u(t)\|_{1}^{2} \equiv$ const, from (3.8) and (3.10) we have

$$
w^{\prime}+K \vartheta(w / K) \leq 0,
$$

or

$$
\int_{0}^{t} \frac{w^{\prime} d t}{K m^{\prime}\left(m^{-1}(w / K)\right)} \geq t .
$$

Since $\left(m^{-1}\right)^{\prime}=1 /\left(m^{\prime}\left(m^{-1}\right)\right)<0$, this leads to the inequality

$$
m^{-1}(w(t) / K)-m^{-1}(w(0) / K) \geq t,
$$

so that $w(t)$ decays like $m(t)$ :

$$
w(t) \leq K m\left(t+m^{-1}(w(0) / K)\right) \leq K m(t) .
$$

In other words, we have $\|u(t)\|_{2} \leq m(t)^{1 / 2}\left\|u_{0}\right\|_{1}$, which is the optimal decay of the $L^{2}$-norm.

REMARK 3.2. If assumptions on $\mathcal{L}$ are as in Section 2 (i.e., stronger than in Theorem 3.2), then $\vartheta$ is a power function (cf. also [16, Th. 6.3]), and in this case the above proof applies to solutions of arbitrary sign (the 
norm $\|u(t)\|_{1}$ might be strictly less than that of $u_{0}$ but this causes no additional difficulties). This remark is essential for the presentation of results concerning solutions of arbitrary sign in Theorem 3.3 below.

A further extension, based on (3.5) and (3.3), of the proof of Theorem 3.2 leads to the estimate

$$
\|u(t)\|_{p} \leq m(t)^{1 / p}\left\|u_{0}\right\|_{p / 2}
$$

for each $2 \leq p<\infty$.

Our next task is to prove the $L^{\infty}$-decay of solutions using a reasoning similar to that in [12, Th. 2.2.7]. Note that, for the conservation laws involving just Brownian diffusion, the authors of [10] found a precise decay in terms of $\left\|u_{0}\right\|_{q}, 1 \leq q \leq p \leq \infty$, including best multiplicative constants, of all $L^{p}$-norms of solutions. They used the generalized logarithmic Sobolev inequalities (equivalent to the Nash inequality), but even for $q=1, p=2$, they needed more restrictive assumptions than those in the proof of the above Theorem 3.2. Also, recall that in [5] we proved the $L^{2}$-decay of solutions of (1.1). Our approach via Fourier splitting was more elementary but it applied only to simpler operators $\mathcal{L}$.

TheOREM 3.3. Suppose that the linear semigroup $e^{-t \mathcal{L}}$ satisfies a decay estimate

$$
\left\|e^{-t \mathcal{L}}\right\|_{2, \infty} \leq e^{M(t)} .
$$

Then solutions of the nonlinear Cauchy problem (1.1)-(1.3) satisfy

$$
\|u(t)\|_{\infty} \leq e^{M(t)}\left\|u_{0}\right\|_{2} .
$$

Proof. Without loss of generality, we may assume that $u(x, t)$ is nonnegative. Indeed, by the comparison principle (cf. Corollary 3.1), we have $|u(x, t)| \leq v(x, t)$ for almost all $x \in \mathbb{R}^{n}$ and $t \geq 0$, where $v(x, t)$ is the solution to (1.1) with initial data $\left|u_{0}(x)\right|$.

Now, it follows from [12, Th. 2.2.4], that (3.11) implies the generalized logarithmic Sobolev inequality (2.5). Here $p=p(s) \in[2, \infty)$ is chosen so that $p(s)=2 t /(t-s), 0 \leq s<t$, hence

$$
\frac{d p}{d s}=\frac{p^{2}}{2 t}, \quad p(0)=2, \quad \lim _{s \rightarrow t} p(s)=\infty,
$$

and

$$
\int_{2}^{\infty} p^{-1} \frac{2 t}{p} d p=t .
$$

We also take $\varepsilon(p)=2 t / p(=t-s)$ in $(2.5)$, and $\Gamma$ becomes $\Gamma(\varepsilon, p)=$ $2 \beta(\varepsilon(p)) / p$ for the function $\beta(\varepsilon)=\Gamma(\varepsilon, 2)$ linked to $(2.5)$. Finally, we define $N$ to have

$$
\frac{d N}{d s}=\frac{2 \beta(\varepsilon(p))}{p \varepsilon(p)} \quad \text { and } \quad N(0)=0 .
$$


Next, we calculate

$$
\begin{aligned}
\frac{d}{d s} \log \left(e^{-N(s)}\|u(s)\|_{p(s)}\right)=\frac{d}{d s}\left(-N+\frac{1}{p} \log \|u\|_{p}^{p}\right) \\
=-\frac{2 \beta(\varepsilon)}{p \varepsilon}-\frac{1}{p^{2}} \frac{p}{\varepsilon} \log \|u\|_{p}^{p} \\
\quad+\frac{1}{p}\|u\|_{p}^{-p}\left(-p\left\langle\mathcal{L} u, u^{p-1}\right\rangle+0+\frac{p}{\varepsilon} \int u^{p} \log u\right) \\
=\frac{1}{\varepsilon}\|u\|_{p}^{-p}\left(\int u^{p} \log u-\varepsilon\left\langle\mathcal{L} u, u^{p-1}\right\rangle-\frac{2 \beta}{p}\|u\|_{p}^{p}-\|u\|_{p}^{p} \log \|u\|_{p}\right) \leq 0
\end{aligned}
$$

in view of (2.5). Observe that

$$
M(t) \equiv \int_{0}^{t} \frac{d N(s)}{s}=\int_{0}^{t} \frac{2 \beta(\varepsilon(p))}{p \varepsilon(p)} d s=\frac{1}{t} \int_{0}^{t} \beta(s) d s,
$$

which gives (3.12). The above argument was adapted from [12, Th. 2.2.7], where it was used in the linear case.

It is immediately seen that under the assumptions of Theorems 3.2 and 3.3 we have

$$
\|u(t)\|_{\infty} \leq e^{M(t / 2)}\|u(t / 2)\|_{2} \leq e^{M(t / 2)} m(t / 2)^{1 / 2}\left\|u_{0}\right\|_{1} .
$$

COROLlary 3.2. Under assumption (2.11) on $e^{-t \mathcal{L}}$, solutions of the initial value problem (1.1)-(1.3) with $u_{0} \in L^{1}\left(\mathbb{R}^{n}\right)$ satisfy the estimates

$$
\|u(t)\|_{p} \leq C_{p} \min \left(t^{-n(1-1 / p) / 2}, t^{-n(1-1 / p) / \alpha}\right)\left\|u_{0}\right\|_{1}
$$

for all $1 \leq p \leq \infty$ and $t>0$. Moreover, if $u_{0} \in L^{1}\left(\mathbb{R}^{n}\right) \cap L^{\infty}\left(\mathbb{R}^{n}\right)$, then

$$
\|u(t)\|_{p} \leq C(1+t)^{-n(1-1 / p) / \alpha},
$$

with a constant $C$ which depends on $\left\|u_{0}\right\|_{1}$ and $\left\|u_{0}\right\|_{p}$.

Proof. Assumption (2.11) on the semigroup generated by $\mathcal{L}$ implies that both functions $e^{M(t)}, m(t)^{1 / 2}$, themselves majorizing the norms $\left\|e^{-t \mathcal{L}}\right\|_{2, \infty}$, $\left\|e^{-t \mathcal{L}}\right\|_{1,2}$, respectively, can be bounded above by $\min \left(c_{1} t^{-n / 4}, c_{2} t^{-n /(2 \alpha)}\right)$. Thus, the interpolation inequality

$$
\|v\|_{p} \leq\|v\|_{1}^{1 / p}\|v\|_{\infty}^{1-1 / p}
$$

and (3.13) give the estimate (3.14), which blows up near $t=0$. The estimate (3.15) results from (3.13) and the $L^{p}$-contraction property (3.3).

REMARK 3.3. An extension

$$
\|u(t)\|_{p} \leq C(1+t)^{-n(1 / q-1 / p) / \alpha}
$$

of (3.15) holds true for solutions of (1.1)-(1.3), all $t \geq 0$, and for the whole range of exponents $1 \leq q \leq 2 \leq p \leq \infty$. Here, the constant $C$ depends on the norms $\left\|u_{0}\right\|_{q}$ and $\left\|u_{0}\right\|_{p}$. To prove this estimate one can modify the proof 
of Theorem 3.3 to have (3.15) for $2 \leq q$ and $p=\infty$. Then, an interpolation concludes the proof.

4. The first order term of asymptotics. Under the decay assumptions on $e^{-t \mathcal{L}}$ formulated in Section 2 we now arrive at a result which gives the first term of the asymptotics of the solution of the problem (1.1)-(1.3). As it happens, that term exactly corresponds to the asymptotics for the solution of the corresponding linear equation with the same initial data. The phenomenon can be viewed as the asymptotic stability of solutions in $L^{1}$, which, in turn, can be interpreted as a mixing property of $e^{-t \mathcal{L}}$, shared by the nonlinear semigroup associated with (1.1) (cf. [22]).

THEOREM 4.1. Assume that $u$ is a solution of the Cauchy problem (1.1)(1.3) with $u_{0} \in L^{1}\left(\mathbb{R}^{n}\right) \cap L^{\infty}\left(\mathbb{R}^{n}\right)$, and that $-\mathcal{L}$ generates the semigroup $e^{-t \mathcal{L}}$ satisfying (2.6)-(2.7) for some $0<\alpha<2$. Furthermore, suppose that $f \in C^{1}\left(\mathbb{R}, \mathbb{R}^{n}\right)$ and $|f(s)| \leq c(R)|s|^{r}$ for some $r>\max ((\alpha-1) / n+1,1)$, a continuous nondecreasing function $c(\cdot)$ on $[0, \infty)$, and $|s| \leq R$. Then, for every $p \in[1, \infty]$,

$$
t^{n(1-1 / p) / \alpha}\left\|u(t)-e^{-t \mathcal{L}} u_{0}\right\|_{p} \rightarrow 0 \quad \text { as } t \rightarrow \infty .
$$

The proof depends on the following

LEMMA 4.1. Under the assumptions of Theorem 4.1, for every $p \in[1, \infty]$, there exists a constant $C$ such that, for all $t>0$,

$$
\begin{aligned}
& \left\|\int_{0}^{t / 2} \nabla \cdot e^{-(t-\tau) \mathcal{L}} f(u(\tau)) d \tau\right\|_{p} \\
& \quad \leq C \begin{cases}t^{-n(1-1 / p) / \alpha-1 / \alpha+1-n(r-1) / \alpha} & \text { for } n(r-1)<\alpha<2, \\
t^{-n(1-1 / p) / \alpha-1 / \alpha} \log (1+t) & \text { for } \alpha=n(r-1), \\
t^{-n(1-1 / p) / \alpha-1 / \alpha} & \text { for } 0<\alpha<n(r-1),\end{cases}
\end{aligned}
$$

and

$$
\begin{aligned}
\left\|\int_{t / 2}^{t} \nabla \cdot e^{-(t-\tau) \mathcal{L}} f(u(\tau)) d \tau\right\|_{p} & \\
& \leq C \begin{cases}t^{-n(1-1 / p) / \alpha-n(r-1) / \alpha-1 / \alpha+1} & \text { for } 1<\alpha<2, \\
t^{-n(1-1 / p) / \alpha-n(r-1) / \alpha} \log (1+t) & \text { for } \alpha=1, \\
t^{-n(1-1 / p) / \alpha-n(r-1) / \alpha} & \text { for } 0<\alpha<1 .\end{cases}
\end{aligned}
$$

Proof. For $\tau \in[0, t / 2]$, we use (2.7), the growth assumptions on $f,(3.7)$ and (3.15), which leads to

$$
\begin{aligned}
\left\|\nabla \cdot e^{-(t-\tau) \mathcal{L}} f(u(\tau))\right\|_{p} & \leq C(t-\tau)^{-n(1-1 / p) / \alpha-1 / \alpha} c\left(\left\|u_{0}\right\|_{\infty}\right)\|u(\tau)\|_{r}^{r} \\
& \leq C(t-\tau)^{-n(1-1 / p) / \alpha-1 / \alpha}(1+\tau)^{-n(r-1) / \alpha}
\end{aligned}
$$


Hence, the $L^{p}$-norm on the left hand side of (4.2) is bounded by

$$
\begin{aligned}
& C \int_{0}^{t / 2}(t-\tau)^{-n(1-1 / p) / \alpha-1 / \alpha}(1+\tau)^{-n(r-1) / \alpha} d \tau \\
& \leq C(t / 2)^{-n(1-1 / p) / \alpha-1 / \alpha} \int_{0}^{t / 2}(1+\tau)^{-n(r-1) / \alpha} d \tau \\
& \leq C \begin{cases}t^{-n(1-1 / p) / \alpha-1 / \alpha+1-n(r-1) / \alpha} & \text { for } n(r-1)<\alpha<2, \\
t^{-n(1-1 / p) / \alpha-1 / \alpha} \log (1+t) & \text { for } \alpha=n(r-1), \\
t^{-n(1-1 / p) / \alpha-1 / \alpha} & \text { for } 0<\alpha<n(r-1) .\end{cases}
\end{aligned}
$$

Using again (2.7) and (3.15), we estimate the $L^{p}$-norm in (4.3) by

$$
\int_{t / 2}^{t}\left\|\nabla e^{-(t-\tau) \mathcal{L}}\right\|_{1,1}\|u(\tau)\|_{r p}^{r} d \tau \leq C(t / 2)^{-n(r-1 / p) / \alpha} \int_{t / 2}^{t}\left\|\nabla e^{-(t-\tau) \mathcal{L}}\right\|_{1,1} d \tau .
$$

Then, for $1<\alpha<2$, we immediately have

$$
\int_{t / 2}^{t}\left\|\nabla e^{-(t-\tau) \mathcal{L}}\right\|_{1,1} d \tau \leq C \int_{t / 2}^{t}(t-\tau)^{-1 / \alpha} d \tau \leq C t^{1-1 / \alpha} .
$$

For $0<\alpha \leq 1$, using (2.7), we obtain

$$
\int_{t / 2}^{t}\left\|\nabla e^{-(t-\tau) \mathcal{L}}\right\|_{1,1} d \tau \leq C \int_{t / 2}^{t-1}(t-\tau)^{-1 / \alpha} d \tau+C \int_{t-1}^{t}(t-\tau)^{-1 / 2} d \tau .
$$

It is easy to see that, if $0<\alpha<1$, both integrals on the right hand side are uniformly bounded for $t \geq 1$. However, for $\alpha=1$, the first of them grows as $\log (1+t)$

Proof of Theorem 4.1. Now, (4.1) is an immediate consequence of the integral equation (3.1), of the decomposition

$$
\int_{0}^{t} \nabla \cdot e^{-(t-\tau) \mathcal{L}} f(u(\tau)) d \tau=\int_{0}^{t / 2} \ldots d \tau+\int_{t / 2}^{t} \ldots d \tau
$$

and of the preceding lemma which gives a more precise decay of the remain$\operatorname{der} u(t)-e^{-t \mathcal{L}} u_{0}$.

5. The second order term of asymptotics. In this section we find the second term of the large time asymptotics of solutions of the problem (1.1)-(1.3). That term reflects nonlinear effects. Compared to the previous sections, the assumptions on the nonlinearity are more restrictive here. For simplicity of exposition, we limit ourselves to the case of $f \in C^{2}\left(\mathbb{R}, \mathbb{R}^{n}\right)$. Since one can always assume that $f(0)=f^{\prime}(0)=0$, we immediately see from the Taylor expansion that $|f(s)| \leq c(R)|s|^{2}$ for $|s| \leq R$ with some continuous 
nondecreasing function $c(\cdot)$ on $[0, \infty)$. Hence, in view of the inequalities $(3.7)$ and (3.15), we have

$$
\int|f(u(y, t))| d y \leq c\left(\left\|u_{0}\right\|_{\infty}\right) \int|u(y, t)|^{2} d y \leq C(1+t)^{-n / \alpha}
$$

Moreover, in the one-dimensional case, the additional assumptions $f \in$ $C^{3}(\mathbb{R}, \mathbb{R})$ and $f^{\prime \prime}(0)=0$ allow us to use the estimate $|f(s)| \leq c(R)|s|^{3}$ for $|s| \leq R$, which implies, in view of (3.3) and (3.15), that

$$
\int|f(u(y, t))| d y \leq c\left(\left\|u_{0}\right\|_{\infty}\right) \int|u(y, t)|^{3} d y \leq C(1+t)^{-2 / \alpha} .
$$

Also, recall that the assumptions (2.8) and (2.9) imposed on the symbol $a(\xi)$ imply the existence of positive constants $C_{1}, C_{2}, C_{3}$ such that

$$
C_{1}|\xi|^{\alpha} \leq a(\xi) \leq C_{2}|\xi|^{\alpha} \quad \text { for }|\xi| \leq 1, \quad C_{3}|\xi|^{2} \leq a(\xi) \quad \text { for }|\xi|>1 .
$$

THEOREM 5.1. Assume that $u$ is a solution of the Cauchy problem (1.1)(1.3) with $u_{0} \in L^{1}\left(\mathbb{R}^{n}\right) \cap L^{\infty}\left(\mathbb{R}^{n}\right)$. Let the semigroup $e^{-t \mathcal{L}}$ satisfy $(2.6)-(2.7)$, and the symbol $a(\xi)$ satisfy the assumptions (2.8)-(2.9) for some $0<\alpha<2$. Suppose that $f \in C^{2}\left(\mathbb{R}, \mathbb{R}^{n}\right), f^{\prime}(0)=0$. If $n=1$ and $\alpha \geq 1$, suppose that $f \in C^{3}(\mathbb{R}, \mathbb{R})$ and $f^{\prime}(0)=f^{\prime \prime}(0)=0$. Then, for each $p \in(1, \infty]$,

$$
\begin{aligned}
& t^{n(1-1 / p) / \alpha+1 / \alpha} \\
& \quad \times\left\|u(t)-e^{-t \mathcal{L}} u_{0}+\left(\int_{0}^{\infty} \int_{\mathbb{R}^{n}} f(u(y, \tau)) d y d \tau\right) \cdot \nabla\left(e^{-t \mathcal{L}}\right)\right\|_{p} \rightarrow 0
\end{aligned}
$$

as $t \rightarrow \infty$.

Proof. First, note that the assumptions on $f$ and (5.1) imply that, for $n \geq 2$ and $0<\alpha<2$, as well as for $n=1$ and $0<\alpha<1$, we have

$$
\int_{0}^{\infty} \int_{\mathbb{R}^{n}}|f(u(y, \tau))| d y d \tau \leq C \int_{0}^{\infty}(1+\tau)^{-n / \alpha} d \tau<\infty .
$$

In view of (5.2), an analogous estimate holds true for $n=1, \alpha \geq 1$, as long as $f^{\prime \prime}(0)=0$.

Now, by the integral representation (3.1) of solutions of (1.1)-(1.3), it suffices to estimate the $L^{p}$-norm of the difference

$$
\int_{0}^{t} \nabla \cdot e^{-(t-\tau) \mathcal{L}} f(u(\tau)) d \tau-\left(\int_{0}^{\infty} \int_{\mathbb{R}^{n}} f(u(y, \tau)) d y d \tau\right) \cdot \nabla\left(e^{-t \mathcal{L}}\right) .
$$

Utilizing (2.7) and (5.5), we immediately obtain

$$
\begin{array}{r}
t^{n(1-1 / p) / \alpha+1 / \alpha}\left\|\left(\int_{t / 2}^{\infty} \int_{\mathbb{R}^{n}} f(u(y, \tau)) d y d \tau\right) \cdot \nabla\left(e^{-t \mathcal{L}}\right)\right\|_{p} \\
\leq C \int_{t / 2}^{\infty} \int_{\mathbb{R}^{n}}|f(u(y, \tau))| d y d \tau \rightarrow 0
\end{array}
$$


as $t \rightarrow \infty$. Moreover, it has already been proved in (4.3) that, for $n \geq 2$ and $0<\alpha<2$ (choose $r=2$ in (4.3)), and for $n=1$ and $\alpha \geq 1$ (choose $r=3$ ),

$$
t^{n(1-1 / p) / \alpha+1 / \alpha}\left\|\int_{t / 2}^{t} \nabla \cdot e^{-(t-\tau) \mathcal{L}} f(u(\tau)) d \tau\right\|_{p} \rightarrow 0
$$

as $t \rightarrow \infty$. To prove this for $n=1$ and $0<\alpha<1$ it suffices to repeat the argument from $[5,(5.9)]$, hence we skip the details. Therefore the proof of (5.4) will have been completed if we can show that, as $t \rightarrow \infty$, the quantity

$$
\begin{aligned}
& t^{n(1-1 / p) / \alpha+1 / \alpha} \\
& \quad \times\left\|\int_{0}^{t / 2} \int_{\mathbb{R}^{n}} \nabla\left(\left(e^{-(t-\tau) \mathcal{L}}\right)(\cdot-y)-\left(e^{-t \mathcal{L}}\right)(\cdot)\right) \cdot f(u(y, \tau)) d y d \tau\right\|_{p}
\end{aligned}
$$

tends to 0 . To prove this assertion, we fix $\delta \in(0,1 / 2)$ and decompose the integration domain $[0, t / 2] \times \mathbb{R}^{n}$ into two parts $\Omega_{1}$ and $\Omega_{2}$, where

$$
\Omega_{1}=[0, \delta t] \times\left\{y \in \mathbb{R}^{n}:|y| \leq \delta t^{1 / \alpha}\right\}, \quad \Omega_{2}=\left([0, t / 2] \times \mathbb{R}^{n}\right) \backslash \Omega_{1} .
$$

We estimate the $L^{p}$-norm of the integral in (5.6) over $\Omega_{2}$ in a straightforward manner by the following quantity:

$$
\begin{aligned}
& \iint_{\Omega_{2}}\left(\left\|\nabla\left(e^{-(t-\tau) \mathcal{L}}\right)(\cdot-y)\right\|_{p}+\left\|\nabla\left(e^{-t L}\right)(\cdot)\right\|_{p}\right)|f(u(y, \tau))| d y d \tau \\
& \leq C\left(\iint_{\Omega_{2}}(t-\tau)^{-n(1-1 / p) / \alpha-1 / \alpha}|f(u(y, \tau))| d y d \tau\right. \\
& \left.+t^{-n(1-1 / p) / \alpha-1 / \alpha} \iint_{\Omega_{2}}|f(u(y, \tau))| d y d \tau\right) \\
& \leq C t^{-n(1-1 / p) / \alpha-1 / \alpha} \iint_{\Omega_{2}}|f(u(y, \tau))| d y d \tau .
\end{aligned}
$$

Now, it easily follows from (5.5) that $\iint_{\Omega_{2}}|f(u(y, \tau))| d y d \tau \rightarrow 0$ as $t \rightarrow \infty$.

Next, we estimate the integral in (5.6) over $\Omega_{1}$. For this purpose, we first derive a suitable bound of

$$
\begin{aligned}
& \sup \left\|\nabla\left(e^{-(t-\tau) \mathcal{L}}\right)(\cdot-y)-\nabla\left(e^{-t \mathcal{L}}\right)(\cdot)\right\|_{p} \\
& |y| \leq \delta t^{1 / \alpha} \\
& 0<\tau \leq \delta t \\
& \begin{aligned}
\leq & \sup _{0<\tau \leq \delta t}\left\|\nabla\left(e^{-(t-\tau) \mathcal{L}}\right)(\cdot)-\nabla\left(e^{-t L}\right)(\cdot)\right\|_{p} \\
& +\sup _{|y| \leq \delta t^{1 / \alpha}}\left\|\nabla\left(e^{-t \mathcal{L}}\right)(\cdot-y)-\nabla\left(e^{-t \mathcal{L}}\right)(\cdot)\right\|_{p} \\
\equiv & I_{1}(\delta, t)+I_{2}(\delta, t)
\end{aligned}
\end{aligned}
$$


for each $p \in[2, \infty], \delta \in(0,1 / 2)$, and $t>0$. The tool here is the HausdorffYoung inequality

$$
\|\widehat{v}\|_{p} \leq C\|v\|_{q},
$$

valid for every $1 \leq q \leq 2 \leq p \leq \infty$ such that $1 / p+1 / q=1$. Recall that

$$
\nabla\left(e^{-t \mathcal{L}}\right)(x)=(2 \pi)^{-n} \int_{\mathbb{R}^{n}} i \xi e^{-t a(\xi)+i x \xi} d \xi,
$$

so, in view of (5.8), we get

$$
\begin{aligned}
\left(I_{1}(\delta, t)\right)^{q} & \leq C \sup _{\tau \leq \delta t} \int_{\mathbb{R}^{n}}|\xi|^{q}\left|e^{-(t-\tau) a(\xi)}-e^{-t a(\xi)}\right|^{q} d \xi \\
& \leq C \sup _{0<\tau \leq \delta t} \int_{|\xi|<1} \ldots d \xi+C \sup _{0<\tau \leq \delta t} \int_{|\xi| \geq 1} \ldots d \xi .
\end{aligned}
$$

Now, using the elementary inequality

$$
\left|e^{-w_{1}}-e^{-w_{2}}\right| \leq\left|w_{1}-w_{2}\right| e^{-w_{2}},
$$

valid for all $0<w_{2} \leq w_{1}$, and the assumptions imposed on $a(\xi)$ (cf. (2.8) and (5.3)), we obtain

$$
\begin{aligned}
\sup _{0<\tau \leq \delta t} \int_{|\xi|<1} \ldots d \xi & \leq \sup _{0<\tau \leq \delta t} \int_{|\xi|<1}|\xi|^{q}|\tau a(\xi)|^{q} e^{-q(t-\tau) a(\xi)} d \xi \\
& \leq C(\delta t)^{q} \int_{|\xi|<1}|\xi|^{q}|\xi|^{q \alpha} e^{-C_{1}(1-\delta) t|\xi|^{\alpha}} d \xi \\
& \leq C \delta^{q} t^{-n / \alpha-q / \alpha}
\end{aligned}
$$

with a constant $C>0$ independent of $\delta$ and $t$. In view of (2.9) and (5.3), the second term on the right hand side of (5.9) is estimated directly by

$$
C \int_{|\xi| \geq 1}|\xi|^{q}\left(e^{-C_{3}(1-\delta) t|\xi|^{2}}+e^{-C_{3} t|\xi|^{2}}\right) d \xi \leq C t^{-N}
$$

for every $N>0$, and a constant $C$ depending on $N$ only.

The reasoning in the case of $I_{2}(\delta, t)$ is similar. Using (5.8) we bound it, for $p \in[2, \infty]$, by

$$
\begin{aligned}
C \sup _{|y| \leq \delta t^{1 / \alpha}} \int_{\mathbb{R}^{n}}|\xi|^{q}\left|e^{i y \xi}-1\right|^{q} e^{-q t a(\xi)} d \xi & \\
& \leq C \sup _{|y| \leq \delta t^{1 / \alpha}} \int_{|\xi|<1} \ldots d \xi+C \sup _{|y| \leq \delta t^{1 / \alpha}} \int_{|\xi| \geq 1} \ldots d \xi .
\end{aligned}
$$

Now, the elementary inequality $\left|e^{i y \xi}-1\right| \leq|y \xi|$ yields

$$
\sup _{|y| \leq \delta t^{1 / \alpha}} \int_{|\xi| \leq 1} \ldots d \xi \leq \sup _{|y| \leq \delta t^{1 / \alpha}} \int_{|\xi| \leq 1}|\xi|^{q}|y \xi|^{q} e^{-C_{1} t|\xi|^{\alpha}} d \xi \leq C \delta^{q} t^{-n / \alpha-q / \alpha}
$$

with a constant $C>0$ independent of $\delta$ and $t$. 
The estimation for the second term on the right hand side of (5.10) is similar to that for the corresponding term in $I_{1}(\delta, t)$, hence we skip the details.

Putting together the above arguments we find that, for each $p \in[2, \infty]$,

$$
\begin{gathered}
|y| \leq \delta t^{1 / \alpha} \\
0<\tau \leq \delta t
\end{gathered}
$$

for every $\delta \in(0,1 / 2), t>0$, and constants independent of $\delta$ and $t$. Applying this estimate to (5.6) we see that the latter is bounded by

$$
\left(C \delta+C t^{-N}\right) \iint_{\Omega_{1}}|f(u(y, \tau))| d y d \tau .
$$

Since $\delta$ was an arbitrary number from $(0,1 / 2)$, in view of $(5.7)$, we conclude that, for every $p \in[2, \infty]$, the quantity in (5.6) tends to 0 as $t \rightarrow \infty$.

To deal with the case $p \in(1,2)$, first observe that (4.2) and (2.7) immediately imply

$$
\begin{aligned}
& \left\|\int_{0}^{t / 2} \int_{\mathbb{R}^{n}}\left(\nabla\left(e^{-(t-\tau) L}\right)(\cdot-y)-\nabla\left(e^{-t \mathcal{L}}\right)(\cdot)\right) \cdot f(u(y, \tau)) d y d \tau\right\|_{1} \\
& \leq\left\|\int_{0}^{t / 2} \int_{\mathbb{R}^{n}} \nabla\left(e^{-(t-\tau) \mathcal{L}}\right)(\cdot-y) \cdot f(u(y, \tau)) d y d \tau\right\|_{1} \\
& \quad+\left\|\nabla\left(e^{-t \mathcal{L}}\right)(\cdot)\right\|_{1} \int_{0}^{\infty} \int_{\mathbb{R}^{n}}|f(u(y, \tau))| d y d \tau \leq C t^{-1 / \alpha} .
\end{aligned}
$$

Now, inequality (3.16), combined with (5.11) and our result for $p=\infty$, gives the proof that, for $1<p<2$, the quantity in (5.6) tends to 0 , as $t \rightarrow \infty$, as well.

REMARK 5.1. Theorem 5.1 does not cover the case $p=1$. However, this missing case can be handled, for $\mathcal{L}$ having the particular form (2.12), by using the approximation of $\left(e^{-t \mathcal{L}}\right)$ by $\left(e^{-t \mathcal{H}}\right)$ for large $t$ developed in $[5$, Lemma 2.2 and Corollary 2.1]; cf. also [13].

REMARK 5.2. Observe that it was crucial in the proof of Theorem 5.1 that $\int_{0}^{\infty} \int_{\mathbb{R}^{n}}|f(u(y, \tau))| d y d \tau<\infty$. Assuming, as in Theorem 4.1, that $|f(s)|$ $\leq c(R)|s|^{r}$ for some $r>\max ((\alpha-1) / n+1,1)$ and $|s| \leq R$, in view of Corollary 3.2 we obtain

$$
\begin{aligned}
\int_{0}^{\infty} \int_{\mathbb{R}^{n}}|f(u(y, \tau))| d y d \tau & \leq C \int_{0}^{\infty} \int_{\mathbb{R}^{n}}|u(y, \tau)|^{r} d y d \tau \\
& \leq C \int_{0}^{\infty}(1+t)^{-n(r-1) / \alpha} d \tau .
\end{aligned}
$$


The integral on the right hand side converges provided $-n(r-1) / \alpha<-1$, i.e., when $r>\alpha / n+1$. This simple observation allows us to relax the conditions imposed on $f$ in Theorem 5.1.

If $\max ((\alpha-1) / n+1,1)<r \leq \alpha / n+1$, the second order term of the asymptotics of solutions of (1.1)-(1.3) differs from that in (5.4); see e.g. [5, Th. 1.2(i)] for $n=1, r=2$. Since the reasoning from [5, Th. 1.2] applies in this case with no essential changes, we will not give the details of calculations (cf. [28] and [17]).

REMARK 5.3. Finally, notice that the case $r=(\alpha-1) / n+1$ is critical in the sense that the asymptotic behavior of solutions of (1.1) is no longer determined by the linear evolution term $e^{-t \mathcal{L}} u_{0}$. Of course, this is not a surprise: for $n=1, \alpha=2, r=2, \mathcal{L}=-\partial^{2} / \partial x^{2},(1.1)$ becomes the classical Burgers equation (1.5) whose solutions have a genuinely nonlinear asymptotic behavior described in Remark 1.3. A candidate self-similar profile, which may describe the asymptotics of solutions in this case, has been constructed for the fractal Burgers equation in [3, Proposition 6.1]. A detailed discussion of this issue for the general problem (1.1)-(1.3) will appear elsewhere (cf. [6]).

Acknowledgements. Part of this research was done while the first and second named authors were research scholars at the CWRU Center for Stochastic and Chaotic Processes in Science and Technology, Cleveland (Summer 1999). Grant support from NSF 97-04231 and KBN 324/P03/97/12, $50 / \mathrm{P} 03 / 2000 / 18$ is also gratefully acknowledged.

\section{References}

[1] C. Bardos, P. Penel, U. Frisch and P. L. Sulem, Modified dissipativity for a nonlinear evolution equation arising in turbulence, Arch. Rational Mech. Anal. 71 (1979), $237-256$.

[2] J. Bertoin, Lévy Processes, Cambridge Univ. Press, 1996.

[3] P. Biler, T. Funaki and W. A. Woyczyński, Fractal Burgers equations, J. Differential Equations 148 (1998), 9-46.

[4] - , - - , Interacting particle approximation for nonlocal quadratic evolution problems, Probab. Math. Statist. 19 (1999), 267-286.

[5] P. Biler, G. Karch and W. A. Woyczyński, Asymptotics for multifractal conservation laws, Studia Math. 135 (1999), 231-252.

[6] -, - - - Critical nonlinearity exponent and self-similar asymptotics for Lévy conservation laws, Ann. Inst. H. Poincaré Anal. Non Linéaire, to appear.

[7] - - - - Multifractal and Levy conservation laws, C. R. Acad. Sci. Paris Sér. I Math. 330 (2000), 343-348.

[8] P. Biler and W. A. Woyczyński, Global and exploding solutions for nonlocal quadratic evolution problems, SIAM J. Appl. Math. 59 (1999), 845-869.

[9] N. Bouleau et F. Hirsch, Formes de Dirichlet générales et densité des variables aléatoires réelles sur l'espace de Wiener, J. Funct. Anal. 69 (1986), 229-259. 
[10] E. A. Carlen and M. Loss, Optimal smoothing and decay estimates for viscously damped conservation laws, with applications to the 2-D Navier-Stokes equation, Duke Math. J. 86 (1996), 135-157.

[11] T. Coulhon, Ultracontractivity and Nash type inequalities, J. Funct. Anal. 141 (1996), 510-539.

[12] E. B. Davies, Heat Kernels and Spectral Theory, Cambridge Univ. Press, 1989.

[13] J. Duoandikoetxea et E. Zuazua, Moments, masses de Dirac et décomposition de fonctions, C. R. Acad. Sci. Paris Sér. I Math. 315 (1992), 693-698.

[14] M. Escobedo and E. Zuazua, Large time behavior for convection-diffusion equations in $\mathbb{R}^{N}$, J. Funct. Anal. 100 (1991), 119-161.

[15] T. Funaki and W. A. Woyczyński, Interacting particle approximation for fractal Burgers equation, in: Stochastic Processes and Related Topics: In Memory of Stamatis Cambanis 1943-1995, I. Karatzas, B. S. Rajput and M. S. Taqqu (eds.), Birkhäuser, Boston, 1998, 141-166.

[16] N. Jacob, Pseudo-Differential Operators and Markov Processes, Akademie-Verlag, Berlin, 1996.

[17] G. Karch, Large-time behavior of solutions to nonlinear wave equations: higher-order asymptotics, Math. Methods Appl. Sci. 22 (1999) 1671-1697.

[18] T. Komatsu, On the martingale problem for generators of stable processes with perturbations, Osaka J. Math. 21 (1984), 113-132.

[19] —, Uniform estimates for fundamental solutions associated with non-local Dirichlet forms, ibid. 32 (1995), 833-860.

[20] O. A. Ladyzhenskaya, V. A. Solonnikov and N. N. Ural'tseva, Linear and Quasilinear Equations of Parabolic Type, Amer. Math. Soc., Providence, 1988.

[21] J. A. Mann Jr. and W. A. Woyczyński, Growing fractal interfaces in the presence of self-similar hopping surface diffusion, Phys. A 291 (2001), 159-183.

[22] R. Rudnicki, Asymptotical stability in $L^{1}$ of parabolic equations, J. Differential Equations 102 (1993), 391-401.

[23] M. F. Shlesinger, G. M. Zaslavsky and U. Frisch (eds.), Lévy Flights and Related Topics in Physics, Lecture Notes in Phys. 450, Springer, Berlin, 1995.

[24] E. M. Stein, Singular Integrals and Differentiability Properties of Functions, Princeton Univ. Press, 1970.

[25] D. W. Stroock, Diffusion processes associated with Lévy generators, Z. Wahrsch. Verw. Gebiete 32 (1975), 209-244.

[26] N. T. Varopoulos, L. Saloff-Coste and T. Coulhon, Analysis and Geometry on Groups, Cambridge Univ. Press, 1992.

[27] W. A. Woyczyński, Burgers-KPZ Turbulence-Göttingen Lectures, Lecture Notes in Math. 1700, Springer, Berlin, 1998.

[28] E. Zuazua, Weakly nonlinear large time behavior in scalar convection-diffusion equations, Differential Integral Equations 6 (1993), 1481-1491.

Instytut Matematyczny

Uniwersytet Wrocławski

Pl. Grunwaldzki 2/4

50-384 Wrocław, Poland

E-mail: biler@math.uni.wroc.pl

karch@math.uni.wroc.pl
Department of Statistics and Center for Stochastic and Chaotic Processes in Science and Technology

Case Western Reserve University Cleveland, OH 44106-7054, U.S.A. E-mail: waw@po.cwru.edu 